\title{
Juvenile idiopathic arthritis and vaccine preventable diseases - a short review
}

\author{
Alexis-Virgil Cochino ${ }^{1,2}$, Oana-Maria Farkas', Sigrid Covaci'1,2 \\ 1"Alessandrescu-Rusescu"National Institute for Mother and Child Health, Bucharest, Romania \\ 2"Carol Davila"University of Medicine and Pharmacy, Bucharest, Romania
}

\begin{abstract}
Juvenile idiopathic arthritis is the most frequent rheumatic disease in pediatrics. Immunosuppressive drugs, either conventional or biologic, used for its treatment, pose higher infectious risks. A short literature review is presented. Even though still safe while being on immunosuppressive drugs, vaccination is advisable to be completed for tuberculosis, hepatitis B, measles, varicella and pneumococcus before such treatment is used. As these vaccines are expensive and at times not available on market, they should all be offered by the national health insurance system to JIA patients.
\end{abstract}

Keywords: juvenile idiopathis arthritis, biologics, vaccine, infection

\section{INTRODUCTION}

Treatment of juvenile idiopathic arthritis (JIA), the most frequent rheumatic disease in pediatrics, comprises a range of inhibitors, affecting either the innate immune system (NSAIDs, steroids, hydroxychloroquine) or the acquired one (methotrexate, azathioprine, cyclophosphamide). Biologic DMARDs were added to this armamentarium in the last two decades, improving disease control, at the cost of heightened infectious risk, especially, but not limited to, TB and viral hepatitis. It has to be noted that JIA, even if untreated, has a higher than normal risk for severe infections (1). Due to interleukin/co-factor blocking these drugs offer, it is feared that infection control might be impaired. Therefore, screening for $\mathrm{TB}$ and viral hepatitis $\mathrm{B}$ and $\mathrm{C}$ is advised before starting the treatment, as well as full vaccination according to national immunisation programmes.

As far as vaccines and JIA are concerned, views may be quite confusing. One one hand, vaccines are traditionally cited as potential triggers for autoimmune diseases. On the other hand, their protection is most welcome in patients under immunosuppression. Nevertheless, live attenuated vaccines are avoided, as there are concerns they may actually cause the respective disease in immunosuppressed patients.
Given all these traditionally accepted but very confusing data, a literature review was undertaken, in order to shed some light on JIA relationship with some of the most frequent and feared infectious diseases.

\section{Viral hepatitis $\mathrm{A}$}

A Pubmed search using [Etanercept "acute hepatitis A"] returned no reports of such association. Furthermore, vaccination seems effective, as more than $90 \%$ of JIA patients vaccinated with HAV achieve seroconversion, with no adverse effects (2). Non-responders were all systemic onset JIA cases with active disease, receiving TNF $\alpha$ blockers.

\section{Viral hepatitis B (VHB)}

This infections is a special case, as most developed countries now vaccinate all infants against VHB. As such, VHB carriers are not so frequent amongst pediatric patients with JIA, and data is extrapolated from adults. A study on 257 adult patients on TNF $\alpha$ blockers showed a $39 \%$ reactivation rate in HBs antigen carriers and only $9 \%$ in those testing positive to anti $\mathrm{HBc}(3)$. While not being associated to adverse effects or JIA reactivation, anti HVB vaccine seems to be less effective in JIA patients (less seroconversion; lower antibodies titre), even if in re- 
mission, effect not influenced by methotrexate or prednisolone treatment $(4,5)$.

\section{Tuberculosis (TB)}

A study of 127 JIA patients on Etanercept, followed for 2 years, showed no TB disease (6). Another nationwide study from Taiwan on 1,495 patients with JIA showed a two-fold increase for TB risk; interestingly, methotrexate treatment was associated to a 5-fold increased risk, while patients on TNF $\alpha$ blockers or without DMARD (either biologic or synthetic) had same risk as general population (7). A study on 167 JIA patients showed a similar to general population risk of latent $\mathrm{TB}$ infection (1.4\%); these patients received chemoprophylaxis and then TNF $\alpha$ blockers for 2 years, none of them developing TB disease (8). There are no data concerning safety of BCG vaccination while on immunosuppressants.

\section{Measles}

There is only one case report of modified measles in an adult treated with Etanercept for rheumatoid arthritis (9). Measles vaccine seems to have the same effectiveness and risks in JIA patients (including patients under Etanercept) as in general population (10-12).

\section{Varicella}

A 10 years study on pediatric patients with autoimmune diseases and developing varicella or herpes zoster, followed at a Swiss centre, found 22 cases (13). Of these, 16 were varicella and presented complications that were classical (cellulitis, sepsis, cerebellitis) albeit quite frequent (25\%). It is noteworthy that the only two vaccinated patients developed no complications. Varicella vaccine seems to be marginally less effective in pediatric patients with autoimmune diseases than in healthy children, according to a study on 25 patients (including JIA); vaccination was not associated with serious side effects or JIA flare (14).

\section{REFERENCES}

1. Walters H.M., Pan N., Lehman T.J.A. et al. A prospective study comparing infection risk and disease activity in children with juvenile idiopathic arthritis treated with and without tumor necrosis factor-alpha inhibitors. Clinical rheumatology. 2015; 34(3):457-464.

2. doi:10.1007/s10067-014-2779-8.

\section{Invasive pneumococcal disease}

Reported cases are all in adult patients; it has to be noted however that they include fatalities. Seven valent conjugated pneumococcal vaccination is followed by seroconversion, albeit with lower titres in some of the TNF $\alpha(50 \%)$ and DMARD $(25 \%)$ treated JIA patients (15). Efficiency of the 23-valent unconjugated vaccine (recommended after 5 years old) seems not to be influenced by TNF $\alpha$ blockade in JIA patients under methotrexate (16). No serious side effects or JIA flare were noted, using either of the aforementioned vaccines.

\section{DISCUSSIONS. CONCLUSIONS}

Being by far the most frequently used biologic DMARD for JIA treatment, most available data are about Etanercept.

Viral hepatitis B, varicella and invasive pneumococcal disease may cause serious problems in a JIA patient being treated with biologic DMARDs. Quite surprisingly, available data suggest methotrexate is the most important risk factor for tuberculosis, and not etanercept. Furthermore, even in cases with latent infection which are to be treated with etanercept, preemptive chemoprophylaxis with isoniazid seems to be very efficient in preventing evolution towards tuberculosis disease. There is no report of severe or complicated viral hepatitis A while on etanercept. Viral hepatitis A and B, measles, varicella and pneumococcal vaccines (both conjugated and unconjugated) seems to be very safe and almost as effective in JIA patients (even if under biologic DMARDs) as in healthy children; there are no such data for BCG vaccination. As such, it is advisable that $\mathrm{BCG}$, viral hepatitis $\mathrm{B}$, measles, varicella and pneumococcal vaccines are completed before starting any type of immunosuppression for JIA, provided their administration is covered by the national health insurance system, as they are expensive. However, with the notable exception of BCG vaccine, they can be still be given while on immunosuppressive drugs, being safe and fairly effective.

Conflict of interest: none declared Financial support: none declared

3. Erguven M., Kaya B., Hamzah O.Y., Tufan F. Evaluation of immune response to hepatitis $A$ vaccination and vaccine safety in juvenile idiopathic arthritis. Journal of the Chinese Medical Association 2011; 74:205-208 
4. Pérez-Alvarez R., Díaz-Lagares C., García-Hernández F. et al. Hepatitis $B$ virus (HBV) reactivation in patients receiving tumor necrosis factor (TNF)-targeted therapy: analysis of 257 cases. Medicine (Baltimore). 2011 Nov; 90(6):359-71. doi: 10.1097/MD. Ob013e3182380a76

5. Kasapcopur O., Cullu F., Kamburothlu-Gokse A. et al. Hepatitis $B$ vaccination in children with juvenile idiopathic arthritis. Annals of the Rheumatic Diseases. 2004; 63(9):1128-1130. doi:10.1136/ ard.2003.013201.

6. Maritsi D., Vartzelis G., Soldatou A., Garoufi A., Spyridis N. Markedly decreased antibody titers against hepatitis B in previously immunised children presenting with juvenile idiopathic arthritis. Clinical and Experimental Rheumatology 2013; 31: 969-973.

7. Constantin T., Foeldvari I., Vojinovic J. et al. Paediatric Rheumatology International Trials Organisation (PRINTO). Twoyear Efficacy and Safety of Etanercept in Pediatric Patients with Extended Oligoarthritis, Enthesitis-related Arthritis, or Psoriatic Arthritis. J Rheumatol. 2016 Apr; 43(4):816-24. doi: 10.3899/ jrheum.150430. Epub 2016 Mar 1.

8. Hsin Y-C., Zhuang L-Z., Yeh K-W., Chang C.W., Horng J-T., Huang J-L. (2015) Risk of Tuberculosis in Children with Juvenile Idiopathic Arthritis: A Nationwide Population-Based Study in Taiwan. PLoS ONE 10(6): e0128768. doi:10.1371/journal. pone.0128768

9. Joan Calzada-Hernández, Jordi Anton-López, Rosa BouTorrent et al. Tuberculosis in pediatric patients treated with antiTNFa drugs: a cohort study. Pediatric Rheumatology 201513:54 DOI: 10.1186/s12969-015-0054-4

10. Takahashi E., Kurosaka D., Yoshida K., Yanagimachi M., Kingetsu I., Yamada A. [Onset of modified measles after etanercept treatment in rheumatoid arthritis]. [Article in Japanese]. Nihon Rinsho Meneki Gakkai Kaishi. 2010; 33(1):37-41.

11. Heijstek M.W., Kamphuis S., Armbrust W., Swart J., Gorter S., de Vries L.D. et al. Effects of the Live Attenuated Measles-
Mumps-Rubella Booster Vaccination on Disease Activity in Patients with Juvenile Idiopathic Arthritis. A Randomized Trial. JAMA 2013; 309(23):2449-2456.

12. Borte S., Liebert U.G., Borte M., Sack U. Efficacy of measles, mumps and rubella revaccination in children with juvenile idiopathic arthritis treated with methotrexate and etanercept. Rheumatology 2009; 48:144-148.

13. Heijstek M.W., Pileggi G.C., Zonneveld-Huijssoon E., Armbrust W., Hoppenreijs E.P., Uiterwaal C.S. et al. Safety of measles, mumps and rubella vaccination in juvenile idiopathic arthritis. Ann Rheum Dis 2007; 66:1384-1387.

14. Raphael Leuvenink, Florence Aeschlimann, Walter Baer et al. Clinical course and therapeutic approach to varicella zoster virus infection in children with rheumatic autoimmune diseases under immunosuppression. Leuvenink et al. Pediatric Rheumatology (2016) 14:34. DOI 10.1186/s12969-016-0095-3

15. Pileggi G.S., de Souza C.B., Ferriani V.P. Safety and Immunogenicity of Varicella Vaccine in Patients with Juvenile Rheumatic Diseases Receiving Methotrexate and Corticosteroids. Arthritis Care \& Research 2010; 62:1034-1039

16. Farmakia E., Kanakoudi-Tsakalidoua F., Spouloub V., Trachanaa M., Pratsidou-Gertsia P., Tritsoni P. et al. The effect of anti-TNF treatment on the immunogenicity and safety of the 7-valent conjugate pneumococcal vaccine in children with juvenile idiopathicarthritis. Vaccine 2010; 28:5109-5113

17. Aikawa N.E., França I.L., Ribeiro A.C., Sallum A.M., Bonfa E., Silva C.A. Short and long-term immunogenicity and safety following the 23-valent polysaccharide pneumococcal vaccine in juvenile idiopathic arthritis patients under conventional DMARDs with or without anti-TNF therapy. Vaccine. 2015 Jan 29; 33(5):6049. doi: 10.1016/j.vaccine.2014.12.030. Epub 2014 Dec 29. 